\title{
On the Use of LoRaWAN for Indoor Industrial IoT Applications
}

\author{
Michele Luvisotto $\mathbb{D}^{1},{ }^{1}$ Federico Tramarin $\mathbb{D}^{2},{ }^{2}$ Lorenzo Vangelista, ${ }^{1}$ and Stefano Vitturi ${ }^{2}$ \\ ${ }^{1}$ Department of Information Engineering, University of Padova, Via Gradenigo 6/B, 35131 Padova, Italy \\ ${ }^{2}$ CNR-IEIIT, National Research Council of Italy, Via Gradenigo 6/B, 35131 Padova, Italy
}

Correspondence should be addressed to Federico Tramarin; federico.tramarin@ieiit.cnr.it

Received 29 January 2018; Accepted 12 April 2018; Published 17 May 2018

Academic Editor: Vicente Casares-Giner

Copyright (C) 2018 Michele Luvisotto et al. This is an open access article distributed under the Creative Commons Attribution License, which permits unrestricted use, distribution, and reproduction in any medium, provided the original work is properly cited.

\begin{abstract}
Low-Power Wide-Area Networks (LPWANs) have recently emerged as appealing communication systems in the context of the Internet of Things (IoT). Particularly, they proved effective in typical IoT applications such as environmental monitoring and smart metering. Such networks, however, have a great potential also in the industrial scenario and, hence, in the context of the Industrial Internet of Things (IIoT), which represents a dramatically growing field of application. In this paper we focus on a specific LPWAN, namely, LoRaWAN, and provide an assessment of its performance for typical IIoT employments such as those represented by indoor industrial monitoring applications. In detail, after a general description of LoRaWAN, we discuss how to set some of its parameters in order to achieve the best performance in the considered industrial scenario. Subsequently we present the outcomes of a performance assessment, based on realistic simulations, aimed at evaluating the behavior of LoRaWAN for industrial monitoring applications. Moreover, the paper proposes a comparison with the IEEE 802.15.4 network protocol, which is often adopted in similar application contexts. The obtained results confirm that LoRaWAN can be considered as a strongly viable opportunity, since it is able to provide high reliability and timeliness, while ensuring very low energy consumption.
\end{abstract}

\section{Introduction and Motivation}

The Internet of things (IoT) concept was introduced towards the turn of the century [1] to indicate an interconnected system of uniquely identifiable objects equipped with radiofrequency identification (RFID) technology. Nowadays the IoT paradigm has expanded, embracing a wide range of communication technologies, software architectures, and applications and can be best defined as "a network of networks where, typically, a massive number of objects/things/sensors/devices are connected through communication and information infrastructures to provide value-added services" [2]. Over the past decade, several IoT solutions have been developed by both industry and academia for various kinds of applications, including smart wearables, smart homes, and smart cities, to name a few [2].

In the next years, the IoT vision is expected to be applied not only to the consumer market but also to productive sectors, dramatically changing manufacturing [3], energy [4], automotive [5], agriculture [6], and other industrial applications, in what has already been termed as "Industrial IoT"
(IIoT). According to a report by the World Economic Forum [7], the IIoT revolution will impact economic sectors that account for nearly two-thirds of the global gross domestic product, changing the basis of competition and redrawing industry boundaries. New connected ecosystems will emerge, allowing significant improvements in operational efficiency as well as the advent of a new outcome economy, where companies no longer deliver products and services, but rather measurable results that create value for their customers [7].

The deployment of IIoT solutions is a complex process that, as addressed in [8] where a functionality-based architecture for IIoT is proposed, impacts on several disciplines, such as communication and computer science.

From a traditional communication aspect, IoT encompasses several heterogeneous systems such as Local Area Networks (LANs), Wireless Sensor Networks (WSNs), cellular networks, mesh, and ad hoc networks, whose interoperability is ensured by the common use of existing Internet protocols, such as the Internet Protocol version 6 (IPv6) [8].

Moving to the industrial scenario, applications often have stringent quality of service (QoS) requirements, in 
terms of robustness, reliability, latency, determinism, energy efficiency, and security $[9,10]$. Therefore, a careful selection of the most appropriate network for a specific application is necessary in order to meet those requirements and provide effective IIoT solutions. Also, according to [11], the true potential of the IIoT paradigm can be unlocked only when a wireless communication architecture is envisioned. As a consequence, it is necessary to analyze the suitability of different wireless networks in view of their deployment in Industrial IoT applications. To this regard, several wireless communication systems have been considered for IoT applications [12]. They range from very short-range solutions such as near field communication (NFC), to extremely long-range ones such as WiMAX and from low-power technologies such as Bluetooth Low Energy (BLE), to high-power ones as cellular networks (2G/3G/4G). The several amendments to the IEEE 802.11 standard for Wireless LANs (WLANs) and to the IEEE 802.15.4 standard for Wireless Personal Area Networks (WPANs) are also highly regarded. Moreover, industrial wireless sensor networks [13, 14] and, more specifically, dedicated wireless networks for industrial applications exist, such as WirelessHART and ISA 100.11a, and are currently being expanded to guarantee IP support under the $6 \mathrm{TiSCH}$ family of standards [15].

In addition to the aforementioned solutions, Low-Power Wide-Area Networks (LPWANs) have recently emerged in the IoT scenario [16], the most popular being NB-IoT, SigFox, Ingenu Weightless, and LoRaWAN. These networks, that are available on licensed as well as unlicensed bands, combine a very long communication range (up to several $\mathrm{kms}$ ) with extremely long battery life, at the cost of a limited throughput. Nowadays, LPWANs are mostly used for outdoor monitoring applications, such as environmental monitoring [17] and smart metering [18]. However, their features are appealing for IIoT applications as well, and hence they have been very recently started to be considered also in this scenario $[11,19]$. Indeed, the significantly high energy efficiency of LPWAN devices can reveal truly interesting for cost-effective IIoT deployments. Moreover, the remarkable communication robustness that allows LPWANs to achieve long-range communications can be useful in industrial applications where the wireless channel is often impaired by multipath and fading [20], thus giving them an edge against other lowpower wireless technologies.

The above considerations represent the main motivation of this paper which, basically, addresses the use of LPWANs, and in particular of LoRaWAN, for indoor industrial monitoring applications and supports this claim with a thorough performance assessment. To this aim, an accurate simulation model for LoRaWAN networks is developed starting from the work in [21], including a realistic channel model for indoor industrial environments.

To the best of the authors' knowledge, this is one of the first works that widely investigates the performance of LoRaWAN for indoor industrial monitoring applications. Indeed, in [22] a case study is presented that addresses a specific indoor LoRaWAN industrial application, whereas in [23] some possible industrial applications of LPWANs are described in general. In [24] indoor applications of
LoRaWAN are considered, but not for industrial scenarios. In [25], other LPWAN solutions are addressed for IIoT and, finally, $[17,21,26]$ deal with the LoRaWAN performance in outdoor scenarios.

In detail, the rest of this paper is organized as follows. Section 2 introduces the most widespread LPWAN solutions, with a particular focus on LoRaWAN. Section 3 describes the scenario considered in this work, namely, indoor industrial monitoring. Section 4 presents a realistic model for LoRaWAN networks employed in industrial environments, followed by a detailed performance analysis in Section 5. Finally, Section 6 concludes the paper and outlines some future directions of research.

\section{Low-Power Wide-Area Networks}

LPWANs are designed to offer affordable connectivity to a high number of low-power devices distributed over large geographical areas. In this section, the most widespread LPWAN solutions are discussed, with a particular focus on LoRaWAN.

2.1. LoRa and LoRaWAN. LoRaWAN is an open network standard [27] developed by the LoRa Alliance, which mainly defines the Medium Access Control (MAC) layer and message formats. It is based on LoRa, a proprietary physical (PHY) layer developed by Semtech Corporation and derived from chirp spread spectrum (CSS) modulation. In this technology, each symbol is spread in a fixed bandwidth, $B$, and the time duration of the symbol is varied according to an index called spreading factor (SF) which can range between 7 and 12. Consequently, the duration of a symbol varies from $1 / B \times$ $2^{7}$ and $1 / B \times 2^{12}$. This spreading technique allows recovering data even when the received power is very low (also under the noise level), thus offering very robust communication, at the cost of a reduced data rate [28], which does not exceed $5.47 \mathrm{Kbps}$. Moreover, transmissions with different spreading factors are somewhat orthogonal to each other, increasing network capacity, as better discussed in Section 4 .

LoRaWAN networks are deployed in the unlicensed industrial, scientific, and medical (ISM) bands of $863-870 \mathrm{MHz}$ in Europe, and $902-928 \mathrm{MHz}$ in the US. According to the regulations, in these bands the transmitting devices must limit their maximum power to $14 \mathrm{dBm}(27 \mathrm{dBm}$ in the 869.4-869.65 MHz sub-band) and adopt either a duty-cycled transmission ( $0.1,1$, or 10 percent according to the sub-band) or a listen-before-talk-adaptive-frequency-agility (LBT/ AFA) behavior.

A LoRaWAN network includes three types of entities, namely, End Devices (EDs), Gateways (GWs), and Network Server (NS). EDs are typical field devices that collect sensor information from the field and, possibly, send commands. They are connected (via wireless links) to one or more GWs that, in turn, are connected (either through a wired or cellular backhaul link) to a single NS, which manages the entire network and originates downlink transmissions (if any). There is no exclusive association between EDs and GWs and the same uplink message can be received by several GWs with different signal qualities. 
The LoRaWAN specifications define three functional classes, namely, Classes A, B, and C, with the first one being mandatory for all LoRaWAN end devices (EDs). Class A EDs access the channel in a random fashion, following an ALOHA-like scheme, and open (at most) two reception windows at predefined slots in time and frequency after each uplink transmission, whereas they remain in sleep mode for the rest of the time. Classes $\mathrm{B}$ and $\mathrm{C}$ devices differentiate mostly for their management of receive windows: class $\mathrm{B}$ EDs can open them at scheduled time intervals (they are synchronized with the NS by means of beacon messages broadcasted by the GWs), while class $\mathrm{C}$ ones keep them always open, clearly sacrificing energy efficiency for low latency. Finally, authentication and encryption mechanisms at different levels (device, network, and application) are envisioned by LoRaWAN specifications to ensure the integrity and security of communications.

2.2. Other LPWAN Solutions. Besides LoRaWAN, several other LPWAN technologies are available in both licensed and unlicensed bands.

The former includes mainly NarrowBand IoT (NBIoT), which is part of 3GPP Release 13. NB-IoT reuses the LTE design, adopting Orthogonal Frequency-Division Multiple Access (OFDMA) for downlink and Single-Carrier Frequency-Division Multiple Access (SC-FDMA) for uplink transmissions, with resource blocks of $15 \mathrm{kHz} \times 0.5 \mathrm{~ms}$ and a maximum bandwidth of $180 \mathrm{kHz}$, offering a peak data rate of $250 \mathrm{Kbps}$. NB-IoT can be deployed in-band, using LTE resources, in the guard band between two LTE bands, or as stand-alone, by replacing a $200 \mathrm{kHz}$ GSM carrier.

In the unlicensed band, the most widespread LPWANs besides LoRaWAN are SigFox, Ingenu, and Weightless. The first one is a proprietary protocol based on ultra narrowband (UNB) modulation, with data rate limited to $100 \mathrm{bps}$ in uplink and 600 bps in downlink. SigFox works in the same bands as LoRaWAN and limits the operation of the connected devices to 140 uplink messages and 4 downlink messages per day. Ingenu is also a proprietary technology which works in the $2.4 \mathrm{GHz}$ band and adopts a patented Random Phase Multiple Access (RPMA) scheme for uplink transmissions, with a maximum data rate of $78 \mathrm{Kbps}$ on 40 different $1 \mathrm{MHz}$ wide channels, each of which can host up to 1200 orthogonal signals, thanks to RPMA. Finally, the Weightless Special Interest Group (SIG) proposes three different standards (Weightless$\mathrm{W}$, Weightless-N, and Weightless-P) deployed in different bands (TV white space and sub-GHz ISM bands), offering different data rates (from $200 \mathrm{bps}$ to $10 \mathrm{Mbps}$ ) and employing different modulation schemes (UNB, Quadrature Amplitude Modulation (QAM) and Phase-Shift Keying (PSK) and different channel access methods (ALOHA, Time-Division Multiple Access (TDMA) and Frequency-Division Multiple Access (FDMA)).

Although all these LPWANs can reveal interesting, in this paper we focus exclusively on LoRaWAN, since it is a really promising and popular network with interesting features. Particularly, (i) it operates in an unlicensed band, (ii) its MAC layer protocol is completely open, and (iii) it can handle an unlimited number of packets.

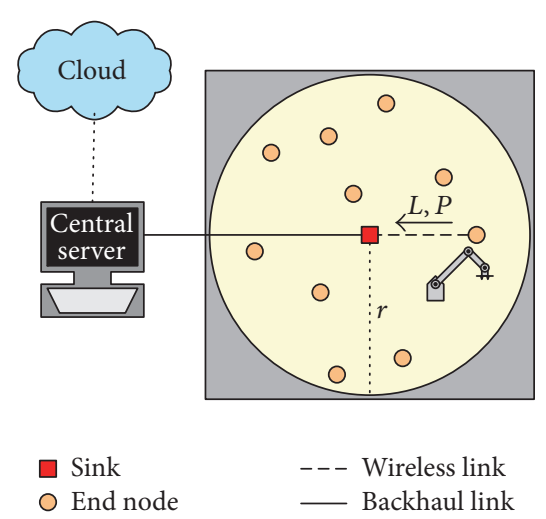

FIGURE 1: A schematic representation of the indoor industrial monitoring scenario considered in this paper.

\section{The Indoor Industrial Monitoring Scenario}

The reference scenario considered in this paper is represented in Figure 1. As can be seen, it refers to a monitoring network composed by $N$ devices (end nodes) deployed in a building where an industrial process is taking place. The devices are distributed within a circular area of radius $r$ and periodically sample different physical quantities that allow monitoring the state of the process. Each node sends the updated sample value as a message of $L$ bytes, with a transmission period of $P$ seconds, to a sink node installed at the center of the building, which will be either a GW in a LoRaWAN network or, more generally, a PAN coordinator in a WPAN. The sink will in turn send the data received from the end nodes to a central server. This configuration resembles that of industrial wireless sensor networks deployed in monitoring systems [29, 30]. Clearly, more complex configurations could be addressed using this type of networks. For example, different applications in which multiple sinks are used could be envisioned, where the nodes can transmit packets of different length with different periods. However, this paper focuses on a basic application of LoRaWAN to indoor IIoT, to investigate its effectiveness, leaving the assessment of more complicated scenarios to future works. For the same reason, we assume that only uplink transmissions are performed and that they are not confirmed, meaning that acknowledgement packets are not sent by the sink nodes and, hence, there are no retransmissions.

Table 1 summarizes the values of the parameters considered in this paper. We assume that the number of nodes in the network ranges from 10 to 1000 , while the coverage radius is fixed to 200 meters. Since sensor measurements typically occupy a few bytes, the message length in these applications is generally low, with 50 bytes being a reasonable value considering that also other information (timestamp, node status, battery life, etc.) may be appended. Finally, the transmission period depends on the dynamics of the sampled quantities and, in this paper, ranges from one minute to 30 minutes. It is worth noting that the corresponding sampling rates are considerably lower than those encountered in other industrial applications (which can be up to some $\mathrm{kHz}$ ), since this scenario is targeted at the on-line monitoring of industrial processes [31], not aiming at real-time control. 
TABLE 1: Parameters for the indoor industrial monitoring scenario.

\begin{tabular}{lcc}
\hline Parameter & Description & Value \\
\hline$N$ & Number of nodes & $\{10, \ldots, 1000\}$ \\
$r$ & Coverage radius & $200 \mathrm{~m}$ \\
$L$ & Message length & 50 Bytes \\
$P$ & Transmission period & $\{60, \ldots, 1800\} \mathrm{s}$ \\
\hline
\end{tabular}

The assessment carried out in this paper is based on three key performance indicators. The first one, related to the communication reliability, is the probability of success (PoS), that is, the percentage of packets sent by the end nodes which are received correctly by the sink. A second important metric, related to the latency and determinism of the communication, is the inter-packet time (IPT), namely, the interval between two consecutively received packets at the sink pertaining to the same node. More formally, if we indicate with $r_{i}(k)$ the time at which the sink receives the $k$ th packet from end node $i, i=1, \ldots, N$, the IPT for node $i$ is the following varying quantity

$$
\operatorname{IPT}_{i}(k)=r_{i}(k+1)-r_{i}(k) .
$$

Considering an observation period during which the sink has received $K_{i}$ packets from node $i$, the mean IPT can be computed as

$$
\operatorname{MIPT}_{i}(k)=\frac{1}{K_{i}-1} \sum_{k=1}^{K_{i}-1} \operatorname{IPT}_{i}(k) .
$$

Then, the global IPT (GIPT) can be computed averaging the MIPT over all the nodes in the network.

The third and last performance metric is the average energy consumed (AEC) by each end node, which provides insights on the energy efficiency and allows forecasting the battery life of end nodes.

\section{A Realistic LoRaWAN Industrial Model}

While it is possible to find accurate models of LoRaWAN networks in the scientific literature [21], none of them tackles the peculiar features of industrial environments. In this section, a realistic model for LoRaWAN networks deployed in IIoT applications is hence presented.

4.1. Channel Model. In this work, indoor LoRaWAN networks deployed in the $863-870 \mathrm{MHz}$ ISM band will be considered, although the channel model is also applicable to the $902-928 \mathrm{MHz}$ ISM band adopted in US. The model should take into account all the impairments that can be present inside industrial buildings, which can be divided into two categories, namely, large-scale effects and small-scale effects.

Large-scale effects include path loss and shadowing, so that the total power loss $L(d)$, is a function of the distance $d$ between transmitter and receiver. It can be expressed (in $\mathrm{dB}$ ) as

$$
L(d)^{\mathrm{dB}}=\mathrm{PL}(d)^{\mathrm{dB}}+\chi_{\sigma}^{\mathrm{dB}}
$$

where the shadowing term, $\chi_{\sigma}^{\mathrm{dB}}$, is generally modeled as a zero-mean Gaussian random variable with standard deviation $\sigma$. The path loss term, $\operatorname{PL}(d)^{\mathrm{dB}}$, instead, is often modeled as a fixed term plus a logarithmic function of the distance $d$ multiplied by a coefficient $\eta$ (path loss exponent), whose value ranges between 2 in ideal conditions (i.e., line-of-sight and free space) and 4 in non-line-of-sight (NLOS) conditions. A common approach is to define a breakpoint distance, referred to as $d_{1}$, beyond which the propagation becomes NLOS, yielding the following path loss model:

$$
\begin{aligned}
& \operatorname{PL}(d)^{\mathrm{dB}} \\
& = \begin{cases}0, & d<d_{0} \\
\mathrm{PL}_{0}+10 \eta_{0} \log \left(\frac{d}{d_{0}}\right), & d_{0} \leq d \leq d_{1} \\
\mathrm{PL}_{0}+10 \eta_{0} \log \left(\frac{d_{1}}{d_{0}}\right)+10 \eta_{1} \log \left(\frac{d}{d_{1}}\right), & d \geq d_{1},\end{cases}
\end{aligned}
$$

where the parameters $\mathrm{PL}_{0}, d_{0}$ and $\eta_{0}$, together with the shadowing standard deviation $\sigma$ can be extracted from [32], which reports real-world measurements in the $900 \mathrm{MHz}$ band from different indoor industrial environments. In this work, the breakpoint distance $d_{1}$ is set to $100 \mathrm{~m}$ (half of the coverage radius) and the path loss exponent in NLOS conditions is set to the typical value $\eta_{1}=4$.

The small-scale effects mostly refer to fading, which is typically modeled through a Rayleigh/Rician distribution, whose only parameter is the $K$-factor $K$. The work in [33] reports some $K$-factor measurements in indoor industrial environments at $868 \mathrm{MHz}$, whose results have been used for the simulations presented in this paper.

4.2. Link Performance Model. Taking into account all channel impairments, the received power at the sink in $\mathrm{dB}$ can be hence modeled as

$$
P_{\mathrm{rx}}^{\mathrm{dBm}}=P_{\mathrm{tx}}^{\mathrm{dBm}}-L^{\mathrm{dB}}-F^{\mathrm{dB}}+G_{\mathrm{tx}}^{\mathrm{dB}}+G_{\mathrm{rx}}^{\mathrm{dB}},
$$

where $P_{\mathrm{tx}}^{\mathrm{dB}}$ is the transmitted power in $\mathrm{dB}, G_{\mathrm{tx}}^{\mathrm{dB}}$ and $G_{\mathrm{rx}}^{\mathrm{dB}}$ are the transmit/receive gains in $\mathrm{dB}$ respectively, $F^{\mathrm{dB}}$ is a margin that accounts for fading, and $L^{\mathrm{dB}}$ is the total loss due to large-scale effects reported in (3). The signal-to-noise ratio (SNR) can be immediately derived from (5) by subtracting the thermal noise power in $\mathrm{dB}$. According to LoRa chipset specifications [34], for each selected spreading factor, $k$, a minimum SNR level $R_{k}$ is requested to achieve a correct demodulation. These values are reported in Table 2, together with the data rate of each SF and the time required to transmit a 50 bytes message (assuming $125 \mathrm{kHz}$ bandwidth and 4/5 code rate).

Besides path loss, shadowing, and fading, the other significant impairment in wireless channels is interference. LoRa CSS modulation is quite robust to external interference from non-LoRa signals [28], whereas the interference between different LoRa transmissions strongly depends on their SF. In [28] it is reported the required signal-to-interference (SIR) ratio in $\mathrm{dB}, T_{k, l}$, to allow a correct decoding of a transmission with SF $k$ when an another transmission with SF $l$ is interfering. However, since in typical LoRaWAN 
TABLE 2: Characteristics of LoRa spreading factors.

\begin{tabular}{lccc}
\hline SF & Required SNR & Data rate & TX time for 50 B \\
\hline 7 & $-7.5 \mathrm{~dB}$ & $5.47 \mathrm{Kbps}$ & $99.58 \mathrm{~ms}$ \\
8 & $-10 \mathrm{~dB}$ & $3.13 \mathrm{Kbps}$ & $178.69 \mathrm{~ms}$ \\
9 & $-12.5 \mathrm{~dB}$ & $1.76 \mathrm{Kbps}$ & $336.90 \mathrm{~ms}$ \\
10 & $-15 \mathrm{~dB}$ & $0.98 \mathrm{Kbps}$ & $632.83 \mathrm{~ms}$ \\
11 & $-17.5 \mathrm{~dB}$ & $0.44 \mathrm{Kbps}$ & $1183.74 \mathrm{~ms}$ \\
12 & $-20 \mathrm{~dB}$ & $0.25 \mathrm{Kbps}$ & $2203.6 \mathrm{~ms}$ \\
\hline
\end{tabular}

networks several concurring transmissions with different SFs are present, evaluating the impact of each interfering SFs may be computationally inefficient. Consequently, an approach based on equivalent signal-to-interference plus noise ratio (ESINR) is detailed in the following.

Consider a transmission with SF $k$ and an interfering transmission with $\mathrm{SF} l$, where the $\mathrm{SIR}$ is $\operatorname{SIR}_{k, l}^{\mathrm{dB}}$. A correct decoding is achieved if $\operatorname{SIR}_{k, l}^{\mathrm{dB}} \geq T_{k, l}$, and also if the SNR is above $R_{k}$. It can be hence said that a SIR level of $T_{k, l}$ is "equivalent" to a SNR level of $R_{k}$ from a system performance perspective. This allows transforming any SIR value with respect to an interfering $S F l$ in an equivalent SIR (ESIR) value as

$$
\mathrm{ESIR}_{k, l}^{\mathrm{dB}}=\operatorname{SIR}_{k, l}^{\mathrm{dB}}+R_{k}-T_{k, l}=\operatorname{SIR}_{k, l}^{\mathrm{dB}}+E_{k, l},
$$

where the matrix $\mathbf{E}$ (in $\mathrm{dB}$ ) is obtained from the values $R_{k}$ in Table 2 and the matrix $T$ in [28] as

$$
\mathbf{E}=\left[\begin{array}{cccccc}
-13.5 & 8.5 & 10.5 & 11.5 & 11.5 & 12.5 \\
14 & -16 & 10 & 12 & 12 & 12 \\
14.5 & 14.5 & -18.5 & 10.5 & 12.5 & 12.5 \\
15 & 15 & 15 & -21 & 11 & 13 \\
15.5 & 15.5 & 15.5 & 15.5 & -23.5 & 11.5 \\
16 & 16 & 16 & 16 & 16 & -26
\end{array}\right]
$$

In other words, (6) allows "normalizing" the SIR between two interfering transmissions with arbitrary spreading factors, transforming it in a quantity comparable with other SNR values and hence allowing to sum them together. This is achieved through the term $E_{k, l}$, which represents a sort of weight indicating the impact of the interfering SF $l$ on the transmission with SF $k$. It is worth observing that this impact is much higher if the two SFs are the same and becomes less relevant as the two SFs are distant, thus allowing concurrent transmissions to take place.

With the proposed model based on ESI, the packet error rate (PER) of a transmission performed with SF $k$ can be evaluated. First, a single transmission is divided into "chunks," each characterized by a specific set of interferers. The bit error rate (BER) during each chunk can be computed through the following operations:

(1) The received power $P_{\mathrm{rx}}^{\mathrm{dBm}}$ is computed according to (5) and the SNR is derived.

(2) The SIR for each interfering SF $l$ is derived and the corresponding ESIR is computed according to (6).

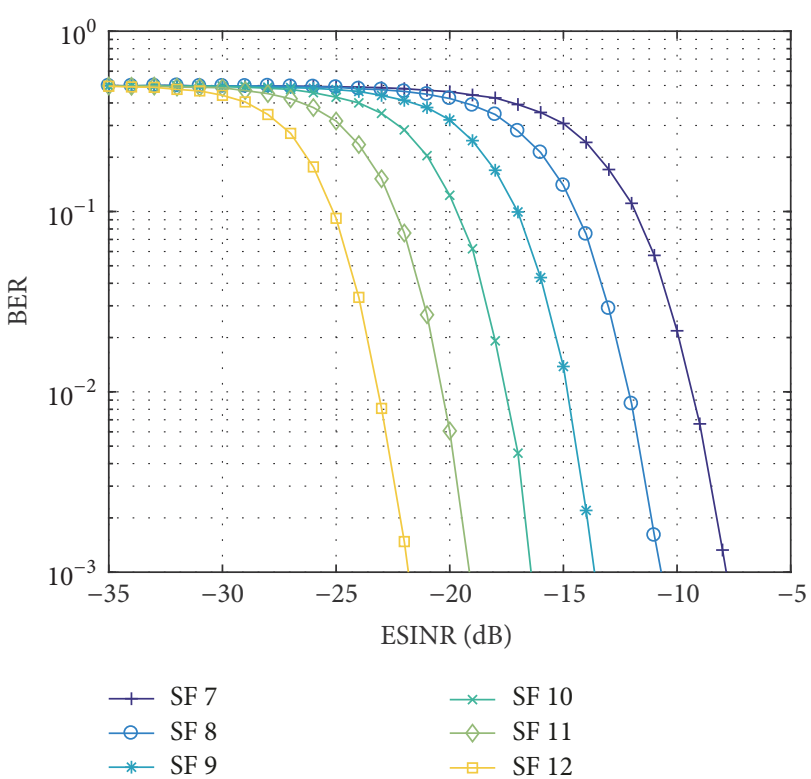

FIgURE 2: BER versus ESINR (in $\mathrm{dB}$ ) curves for different LoRa spreading factors obtained through Matlab simulations.

(3) The ESINR in dB is computed as

$$
\operatorname{ESINR}_{k}^{\mathrm{dB}}=-10 \log _{10}\left(10^{-\mathrm{SNR}_{k}^{\mathrm{dB}} / 10}+\sum_{l=7}^{12} 10^{-\mathrm{ESIR}_{k, l}^{\mathrm{dB}} / 10}\right) .
$$

(4) The BER corresponding to the ESINR is derived according to the curves in Figure 2, which are obtained through Matlab simulations of CSS performance.

Starting from the BER and the number of bits in the chunk, which can be approximated from the chunk duration and the data rate, the chunk error rate (CER) is obtained. Finally, the PER can be derived as

$$
\operatorname{PER}_{k}=1-\prod_{n=1}^{N_{\mathrm{cks}}}\left(1-\mathrm{CER}_{k, n}\right)
$$

where $N_{\text {cks }}$ is the number of chunks that make up the transmission.

4.3. Strategies for the Choice of the Spreading Factors. One of the degrees of freedom in configuring a LoRaWAN network is to assign to each node its SF. In this paper we address some techniques that allow a static assignment of the SFs, as described below. It is worth mentioning, however, that the standard also allows for a dynamic strategy, called adaptive data rate $(\mathrm{ADR})$, which is not considered in this paper since it requires downlink transmissions. Nonetheless, the techniques that are going to be presented can be easily extended to the dynamic case.

The simplest approaches are either to assign the same SF to all the nodes in the network, or to randomly distribute all the available SFs among them. A more refined approach, 
often adopted in other studies [21], is to assign to each node the lowest SF for which the SNR at the sink is higher than the threshold defined in Table 2. However, as discussed in [35], when the maximum distance between nodes and sink is limited (as in the case of indoor environments), this strategy will always lead to all the nodes being assigned the lowest SF, that is, 7 (which ensures the highest data rate), thus incurring in fairness problems and not fully exploiting the orthogonality of the different LoRa SFs.

To overcome this issue, an innovative strategy for the selection of SFs is proposed in this paper, based on a constrained optimization procedure. Let $\mathcal{S}$ be the set of available SFs and $s_{i}$ the SF assigned to node $i$. This procedure needs to fulfill two constraints:

(a) The aforementioned constraint on the SNR, that is,

$$
\mathrm{SNR}_{i}^{\mathrm{dB}} \geq R_{s_{i}}, \quad i=1, \ldots, N
$$

(b) An additional constraint on the transmit period $P$, that is,

$$
P \geq \frac{\operatorname{TX}\left(s_{i}\right)}{\mathrm{DC}_{i}}, \quad i=1, \ldots, N
$$

where $\operatorname{TX}\left(s_{i}\right)$ is the transmission time for SF $i$ as reported in Table 2 and $\mathrm{DC}_{i}$ is the duty cycle limitation of node $i$, which depends on the operating frequency band [36].

After ensuring that these constraints are observed, the SFs are distributed in the most uniform possible way among the nodes, to maximize the orthogonality of transmissions. More formally, let $\mathcal{N}_{k}$ be the set of nodes for which SF $k$ is assigned and let $N_{\min }$ and $N_{\max }$ be the minimum and maximum cardinality of the sets $\mathcal{N}_{k}$, that is,

$$
\begin{aligned}
& N_{\text {min }}=\min _{k \in \mathcal{S}}\left|\mathcal{N}_{k}\right|, \\
& N_{\max }=\max _{k \in \mathcal{S}}\left|\mathcal{N}_{k}\right| .
\end{aligned}
$$

For each node, a SF is chosen among the ones that respect the constraints of both (10) and (11), so that the difference between the minimum and maximum cardinality is minimized:

$$
\arg \min \left|N_{\max }-N_{\min }\right| \text {. }
$$

This strategy, which is indicated as "fair" in the following section, ensures that orthogonality of transmissions is maximized and that the nodes never exceed the duty cycle limitations.

4.4. Energy Model. A fundamental aspect to address in a LoRaWAN network is the energy consumed by the end nodes. A LoRa ED can be in four possible states [34]: idle, standby, transmitting, and receiving. Specifically, a node is always in idle state except when it transmits a packet and during the automatically opened receive windows, where it can be either
TABLE 3: Supply current for LoRa EDs in different states.

\begin{tabular}{lcc}
\hline State & Symbol & Value \\
\hline Idle & $I_{\text {idle }}$ & $1.5 \mu \mathrm{A}$ \\
Standby & $I_{\text {standby }}$ & $1.4 \mathrm{~mA}$ \\
$\mathrm{Tx}$ & $I_{\mathrm{tx}}$ & $28 \mathrm{~mA}$ \\
$\mathrm{Rx}$ & $I_{\mathrm{rx}}$ & $11.2 \mathrm{~mA}$ \\
\hline
\end{tabular}

standby or receiving (not in the case of this paper, since there are no downlink transmissions).

Table 3 reports the current consumed by a LoRa ED in the four different states, as reported in [34]. In order to compute the energy consumption of each ED, the current of each state has been multiplied by the supply voltage $\left(V_{\mathrm{DC}}=3.3 \mathrm{~V}\right)$ and by the time that the ED passes in that specific state. For example, the energy consumed by a single node during a transmission period, to transmit a payload of $50 \mathrm{~B}$ with spreading factor $s$, is

$$
\begin{aligned}
\mathrm{EC}= & V_{\mathrm{DC}} \cdot I_{\mathrm{idle}} \cdot\left[P-\mathrm{TX}(s)-T_{\mathrm{RX}, 1}-T_{\mathrm{RX}, 2}\right]+V_{\mathrm{DC}} \\
& \cdot I_{\mathrm{tx}} \cdot \mathrm{TX}(s)+V_{\mathrm{DC}} \cdot I_{\mathrm{rx}}\left(T_{\mathrm{RX}, 1}+T_{\mathrm{RX}, 2}\right),
\end{aligned}
$$

where $T_{\mathrm{RX}, 1}$ and $T_{\mathrm{RX}, 2}$ are the durations of the first and second receive windows, respectively.

\section{Performance Evaluation}

The LoRaWAN industrial model presented in Section 4 has been implemented in the popular ns3 network simulator [37] in order to assess the performance of this network in the IIoT scenario.

5.1. Simulations Setup. The starting point for the development of ns3 LoRaWAN simulations was the work in [21], in which an original LoRa module for ns3 was presented, allowing accurate simulation of uplink transmission in a LoRaWAN network. Several features have been integrated to this module to allow realistic simulations of LoRaWAN networks employed in IIoT applications. Specifically:

(i) An accurate channel model for indoor industrial buildings has been introduced that accounts for path loss, shadowing, and fading. Realistic channel model parameters were selected considering the experimental measurements reported in [32, 33].

(ii) The simplified link performance model of [21] has been expanded, adding the ESINR-based approach detailed in Section 4.2 as well as the Matlab-based BER versus ESINR curves.

(iii) A different interference model with respect to [21] has been used: in the old model, the power of a partially interfering transmission was "equalized" on the entire packet duration, whereas, as discussed in Section 4 , in the adopted model a packet is divided into chunks, each one with a specific set of interferers and corresponding error rate. 


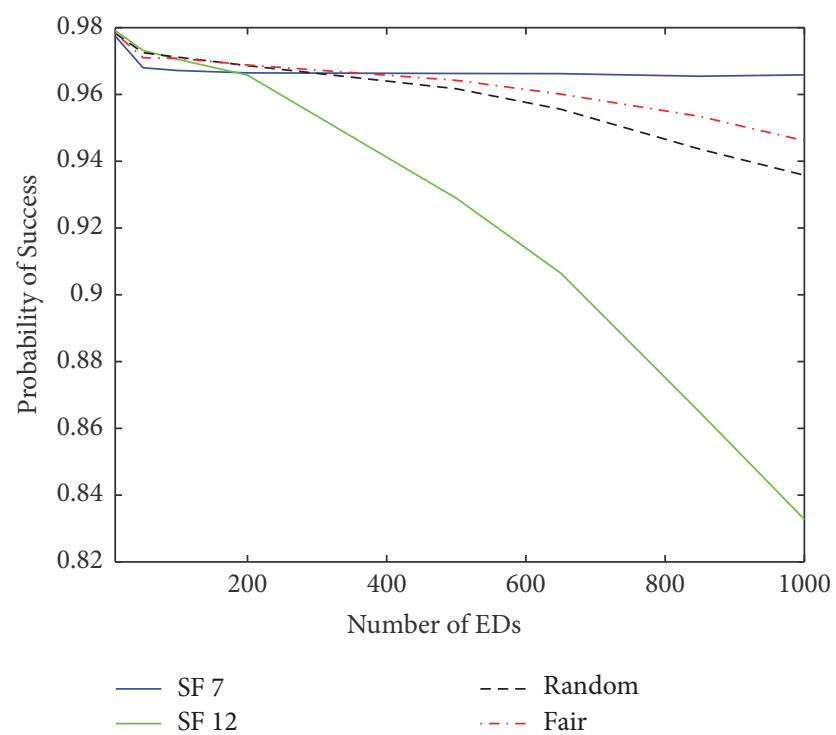

FIgURE 3: PoS versus number of EDs in a LoRaWAN network employed in IIoT applications for different SF choice strategies. The transmission period is fixed to 10 minutes.

(iv) An energy model has been added to [21], allowing a precise computation of the energy consumed by the end nodes.

The performance figures of LoRaWAN have been also compared with those of a WPAN, which have been simulated exploiting the $1 r$-wpan module included in the standard ns3 distribution, upgraded with some modifications. In particular, the $868 \mathrm{MHz}$ binary phase-shift keying (BPSK) PHY layer introduced in the IEEE 802.15.4-2006 standard [38] was considered, in order to provide a fair comparison with LoRaWAN, deployed in the same frequency band. This choice limited the achievable data rate of IEEE 802.15.4 to $20 \mathrm{Kbps}$. Moreover, the modifications proposed in [39], mostly concerning the introduction of an energy model, have been considered.

In all the simulations the reference scenario is that reported in Figure 1, with the parameters shown in Table 1. For each choice of parameters, the results were averaged over 10 different runs, each simulating the network performance for 2 hours.

5.2. Tuning of a LoRaWAN Network. A first assessment is concerned with the comparison of different strategies for the assignment of SFs in a LoRaWAN network used for indoor industrial monitoring applications. Four different strategies, defined in agreement with the analysis carried out in Section 4.3, have been considered, namely, two constant strategies in which all the nodes are assigned either the lowest spreading factor (SF 7) or the highest (SF 12); a random strategy in which SFs are randomly assigned to the nodes; the innovative strategy described in Section 4.3, called "fair."

Figure 3 reports the PoS, computed over all the transmissions in the network, for the different SF assignment strategies and for different networks sizes, ranging from 10

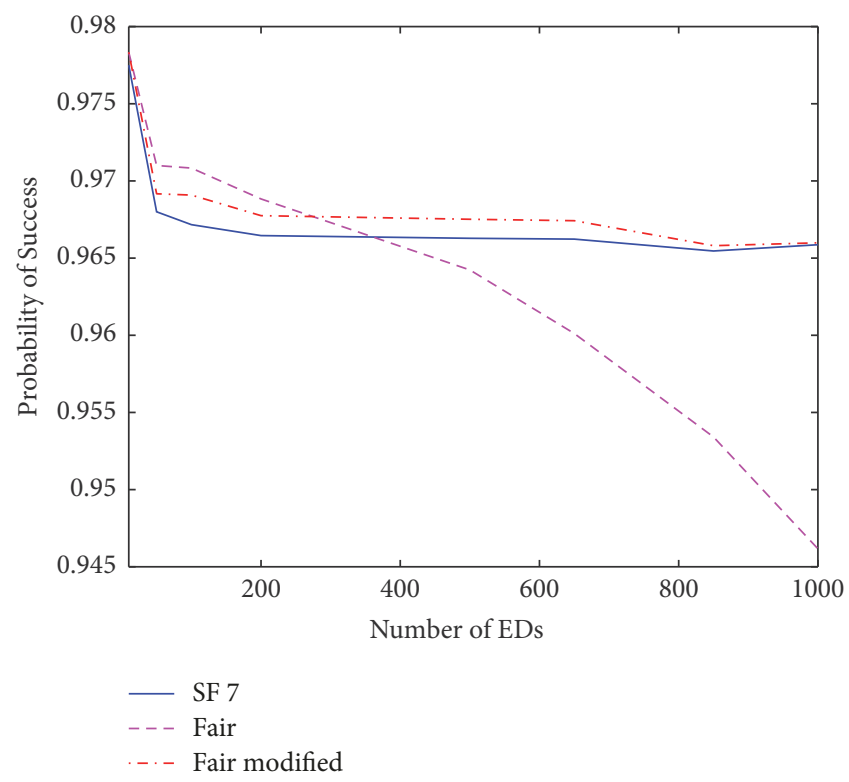

FIgURE 4: Detailed comparison of different SF choice strategies in a LoRaWAN: constant to SF 7, fair selection on the complete list of SFs and fair selection on a restricted list (SF 7, 8 and 9).

to 1000 nodes. The transmission period for all nodes is fixed to 10 minutes. It can be observed that, first, the PoS is generally high, confirming the good robustness of LoRa modulation. The worst behavior is obtained when all nodes are assigned SF 12, especially as the network size increases, since the transmission time with this SF is quite long (more than 2 seconds per packet) and causes severe interference problems. Conversely, when all nodes are assigned SF 7, the PoS is almost constant to a very high value (about 97\%), performing even better (when the number of nodes in the network is high) than the "fair" strategy, which instead yields a slightly higher PoS when there are less than 200 nodes. This result can be explained observing that SF 7 guarantees the lowest transmission time, thus minimizing the interference; conversely, the "fair" strategy leverages the orthogonality of different SFs, but distributes all the available SF values (including the highest ones) among the nodes, increasing the transmission times, and hence ultimately increasing the probability a transmission is subjected to interference.

To overcome this issue, a slightly modified version of the fair strategy is proposed, in which the set of available SFs $\mathcal{S}$ is limited to the three lowest ones $(7,8$, and 9$)$. The performance of this upgraded scheme can be observed in Figure 4, always relevant to $\mathrm{PoS}$ versus number of EDs with $P=10$ minutes. The modified fair strategy now outperforms the strategy in which all nodes are assigned SF 7 both when the number of nodes is low and when it is high, never falling below a PoS of $96.5 \%$. As a result of these considerations, in the following of this paper, the only considered schemes for the selection of SFs in LoRaWAN are the "fair modified" strategy and the "constant to SF 7" one.

5.3. Comparison of LoRaWAN and IEEE 802.15.4. To support the claim that LoRaWAN can represent a good choice for IIoT 


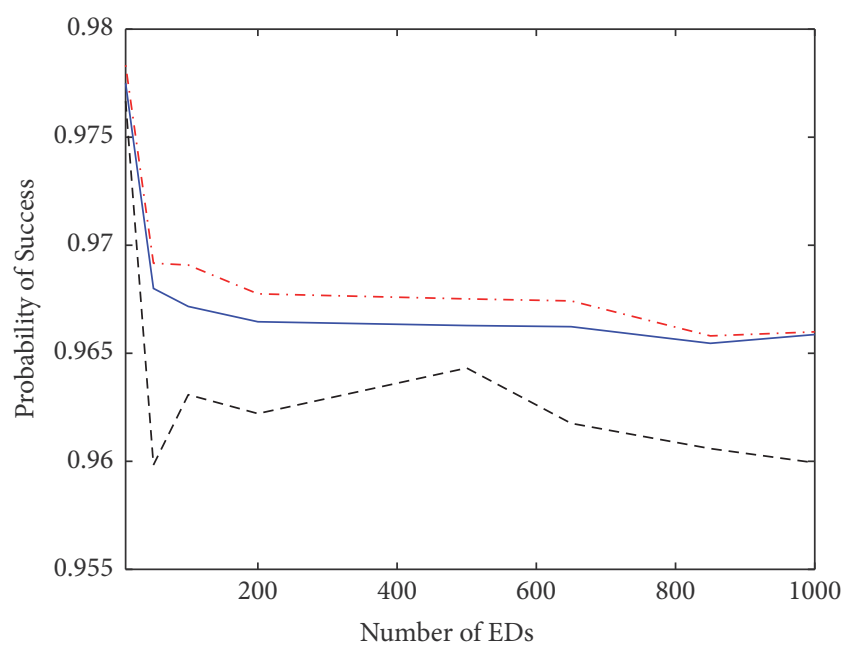

— LoRaWAN SF 7

- - - LoRaWAN Fair Mod.

- - - IEEE 802.15.4

FIGURE 5: PoS versus number of nodes for LoRaWAN and IEEE 802.15.4 employed in IIoT applications. The transmission period is fixed to 10 minutes.

monitoring applications, the performance of this network is compared with that of an IEEE 802.15.4-based one.

The configuration of the IEEE 802.15.4 network is equivalent to that of LoRaWAN. Moreover, the non-beacon version of the IEEE 802.15.4 protocol has been considered, in which nodes (that generate new packets with period $P$ ) access the channel in a random fashion, following a carrier sense multiple access (CSMA) algorithm.

The first comparison, shown in Figure 5, reports the PoS for different network sizes and a transmission period fixed to 10 minutes. It can be observed that both LoRaWAN strategies ("constant to SF 7" and "fair modified") slightly outperform IEEE 802.15.4. This is due to the better robustness of LoRa modulation with respect to the IEEE 802.15.4 one; indeed, comparing the derivations reported in the standard [38] with the curves in Figure 2, IEEE 802.15.4 needs almost a $10 \mathrm{~dB}$ higher SNR to offer the same BER as the less robust LoRa SF (the BER of IEEE 802.15.4 with the $868 \mathrm{MHz}$ BPSK PHY has been derived from the standard [38]). Moreover, in LoRaWAN the collision probability is reduced with respect to IEEE 802.15 .4 because of the orthogonality between different SFs.

A second metric considered in the comparison between LoRaWAN and IEEE 802.15.4 is the GIPT, namely, the time elapsed between two consecutive correct packets received at the sink averaged over all the nodes in the network. A performance assessment under the same conditions of Figure 5 ( $N$ ranging from 10 to 1000 and $P=10$ minutes) is provided in Figure 6. It can be observed that all values are quite close to the transmission period, as expected, even if, again, IEEE 802.15.4 performs slightly worse than LoRaWAN for every network size. This is due to both the lower robustness of IEEE 802.15.4 modulation and the higher randomness of its channel access scheme. Among the two

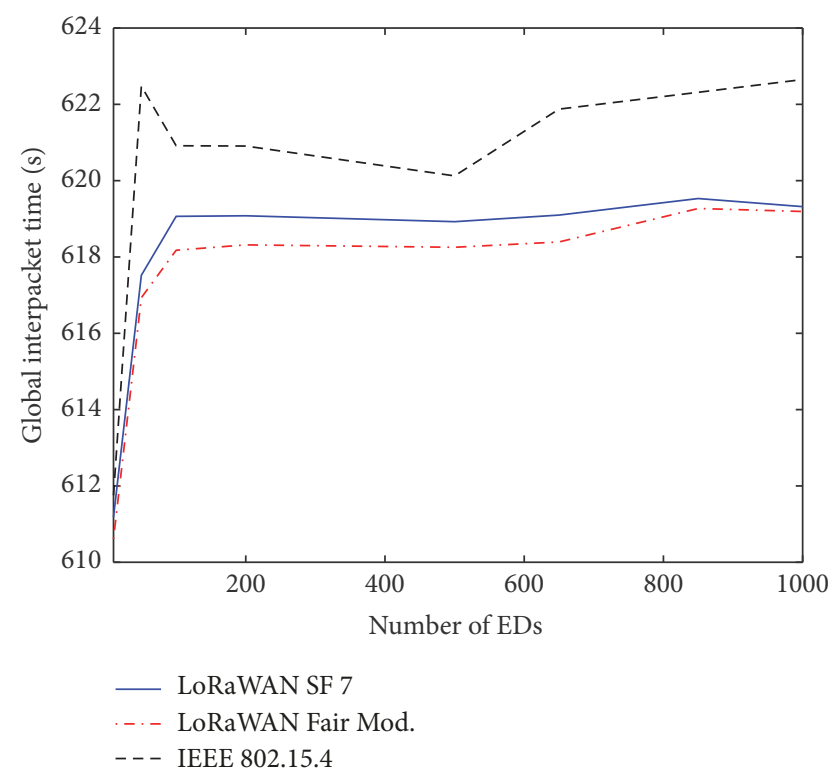

FIGURE 6: GIPT versus number of nodes for LoRaWAN and IEEE 802.15.4 employed in IIoT applications. The transmission period is fixed to 10 minutes.

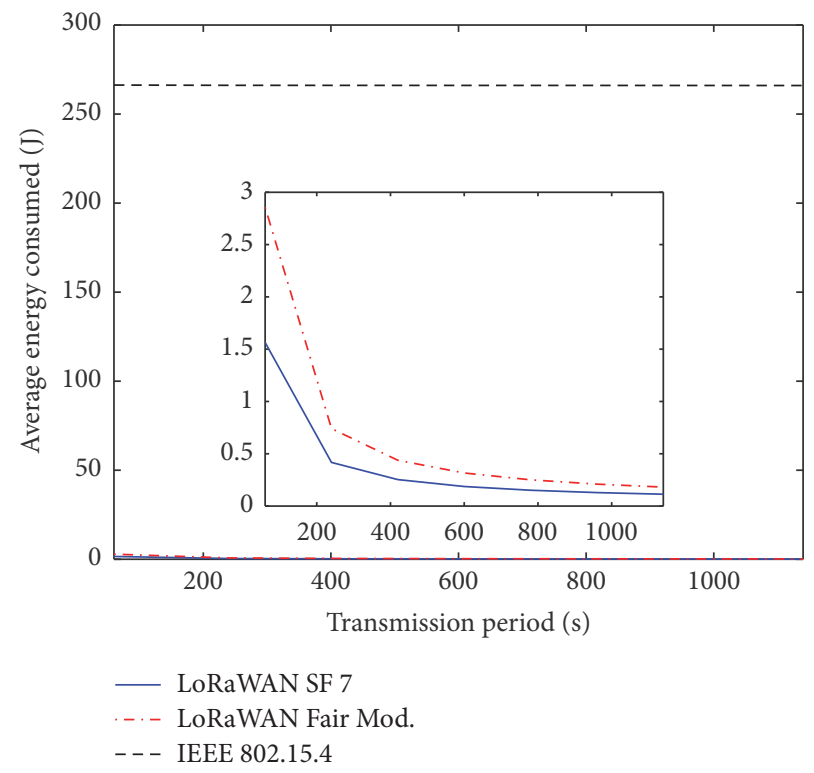

FIGURE 7: Average energy consumed in a 2-hours simulation versus transmission period for LoRaWAN and IEEE 802.15.4 employed in IIoT applications. The number of nodes is fixed to 500. The detailed plot allows to better distinguish the performance of the two SF choice strategies adopted in LoRaWAN.

LoRaWAN strategies, the "fair modified" strategy confirms to be the best one, offering a slightly lower GIPT.

Finally, the performance figures of LoRaWAN and IEEE 802.15.4 are compared in terms of the average energy consumed by each node in Figure 7. In this case the number of nodes is fixed to 500 and the transmission period is varied from 1 to 30 minutes. First, it can be observed that the average energy consumed by IEEE 802.15.4 nodes is 
roughly 100 times higher than that of LoRaWAN nodes, which stay most of the time in deep sleep state and hence have an extremely low-power consumption. To this regard, it is worth observing that the IEEE 802.15.4 protocol has been implemented according to its legacy version, without adopting any specify power saving strategy. Clearly, the introduction of (purposely defined) strategies of this type could definitely lead to better performance in terms of power consumption [40].

The detailed plot in Figure 7 allows also comparing the two LoRaWAN strategies for SF choice, "constant to SF 7" and "fair modified," showing that the former scheme allows consuming less energy. This is motivated by the fact that the energy consumed is directly proportional to the amount of time spent in TX state, which drains the highest current as reported in Table 3, and SF 7 offers the lowest possible transmission time. However, it has to be considered that, as shown in Figure 4, setting the spreading factors of all nodes to 7 also yields a higher probability of error. Consequently, if retransmissions were activated, the additional attempts necessary to transmit corrupted packets would certainly increase the energy consumption. In any case it is worth observing that, from the simulations carried out, the estimation of the battery lifetime for an ED results in about 2 years for a simple 1000 mAh battery.

It has to be noted that the nonbeacon version of IEEE 802.15.4 considered in this section is only one of the many options provided by this standard, which recently defined new operating modes specifically tailored for the monitoring and control of industrial processes, such as the Time-Slotted Channel Hopping (TSCH) mode of IEEE 802.15.4e [41]. These enhancements allow achieving a much improved timeliness, reliability, and energy consumption with respect to the nonbeacon version of the standard and are definitely to be preferred when performing control of critical processes. Nonetheless, in many noncritical industrial monitoring applications such as the one discussed in this paper, where sampling rates are not very high, LoRaWAN can provide a cost-effective and easy-to-deploy alternative, which still guarantees very high reliability and ultralow energy consumption, as proven by the simulation results.

\section{Conclusions}

This paper addressed the adoption of LoRaWAN for indoor industrial monitoring systems which represent an interesting IIoT field of application. After an accurate theoretical analysis, a realistic simulation model of LoRaWAN has been developed that allowed investigating the behavior of network configurations typically deployed for industrial monitoring. The obtained results showed very good performance in terms of reliability, timeliness, and energy consumption. Particularly, a newly introduced technique for the selection of the spreading factor revealed able to outperform other traditionally adopted techniques. A comparison with a configuration of an equivalent WPAN, namely, IEEE 802.15.4, has also been carried out and provided encouraging results.

Several future activities can be envisioned as follow-ups of this work. First, the occurrence of downlink transmissions has to be appropriately investigated in order to evaluate the performance of LoRaWAN in presence of retransmissions and adaptive data rate strategies. It would be also important to model Class B LoRa devices, as they support synchronization through beacons, and, hence, may allow developing a scheduled channel access method (e.g., TDMA) which can improve timeliness. Moreover, in LoRaWAN networks the EDs do not have direct Internet connectivity, so that they can be only remotely accessed through GWs. To this regard, some proposals to integrate IPv6 over LoRaWAN have been already developed [42]: their feasibility and impact on network performance, however, need to be carefully investigated.

Finally, a further step of analysis is represented by the execution of experimental sessions on real LoRaWAN testbeds that would allow tuning the accuracy of the theoretical and simulation analyses presented in this work.

\section{Data Availability}

We have not yet made the data publicly available because some parts of the code are protected.

\section{Conflicts of Interest}

The authors declare that they have no conflicts of interest.

\section{References}

[1] K. Ashton, “That 'Internet of Things' Thing," http://www.rfidjournal.com/articles/view? 4986 .

[2] C. Perera, C. H. Liu, and S. Jayawardena, "The emerging internet of things marketplace from an industrial perspective: a survey," IEEE Transactions on Emerging Topics in Computing, vol. 3, no. 4, pp. 585-598, 2015.

[3] F. Tao, Y. Zuo, L. D. Xu, and L. Zhang, "IoT-based intelligent perception and access of manufacturing resource toward cloud manufacturing," IEEE Transactions on Industrial Informatics, vol. 10, no. 2, pp. 1547-1557, 2014.

[4] S. Jacobsson and A. Bergek, "Transforming the energy sector: The evolution of technological systems in renewable energy technology," Industrial and Corporate Change, vol. 13, no. 5, pp. 815-849, 2004.

[5] L. L. Bello, "Novel trends in automotive networks: A perspective on Ethernet and the IEEE Audio Video Bridging," in Proceedings of the 2014 IEEE Emerging Technology and Factory Automation (ETFA), pp. 1-8, Barcelona, Spain, September 2014.

[6] F. TongKe, "Smart Agriculture Based on Cloud Computing and IOT," Journal of Convergence Information Technology, vol. 8, no. 2, pp. 210-216, 2013.

[7] A. Hassanzadeh, S. Modi, and S. Mulchandani, "Towards effective security control assignment in the Industrial Internet of Things," in Proceedings of the 2015 IEEE 2nd World Forum on Internet of Things (WF-IoT), pp. 795-800, Milan, Italy, December 2015.

[8] L. D. Xu, W. He, and S. Li, "Internet of things in industries: a survey," IEEE Transactions on Industrial Informatics, vol. 10, no. 4, pp. 2233-2243, 2014.

[9] T. Sauter, "The three generations of field-level networks - Evolution and compatibility issues," IEEE Transactions on Industrial Electronics, vol. 57, no. 11, pp. 3585-3595, 2010. 
[10] E. Toscano and L. Lo Bello, "A topology management protocol with bounded delay for Wireless Sensor Networks," in Proceedings of the Factory Automation (ETFA 2008), pp. 942-951, Hamburg, Germany, September 2008.

[11] S. Mumtaz, A. Alsohaily, Z. Pang, A. Rayes, K. F. Tsang, and J. Rodriguez, "Massive Internet of Things for Industrial Applications: Addressing Wireless IIoT Connectivity Challenges and Ecosystem Fragmentation," IEEE Industrial Electronics Magazine, vol. 11, no. 1, pp. 28-33, 2017.

[12] S. Tayeb, S. Latifi, and Y. Kim, "A survey on IoT communication and computation frameworks: An industrial perspective," in Proceedings of the 7th IEEE Annual Computing and Communication Workshop and Conference, CCWC 2017, January 2017.

[13] V. C. Gungor and G. P. Hancke, "Industrial wireless sensor networks: challenges, design principles, and technical approaches," IEEE Transactions on Industrial Electronics, vol. 56, no. 10, pp. 4258-4265, 2009.

[14] K. Beom-Su, v. HoSung, K. K. Hoon, D. Godfrey, and K. KiIl, "A survey on real-time communications in wireless sensor networks," Wireless Communications and Mobile Computing, vol. 2017, Article ID 1864847, 14 pages, 2017.

[15] T. Watteyne, V. Handziski, X. Vilajosana et al., "Industrial Wireless IP-Based Cyber-Physical Systems," Proceedings of the IEEE, vol. 104, no. 5, pp. 1025-1038, 2016.

[16] U. Raza, P. Kulkarni, and M. Sooriyabandara, "Low Power Wide Area Networks: An Overview," IEEE Communications Surveys \& Tutorials, vol. 19, no. 2, pp. 855-873, 2017.

[17] W. Guibene, J. Nowack, N. Chalikias, K. Fitzgibbon, M. Kelly, and D. Prendergast, "Evaluation of LPWAN Technologies for Smart Cities: River Monitoring Use-Case," in Proceedings of the 2017 IEEE Wireless Communications and Networking Conference Workshops, WCNCW 2017, March 2017.

[18] N. Varsier and J. Schwoerer, "Capacity limits of LoRaWAN technology for smart metering applications," in Proceedings of the 2017 IEEE International Conference on Communications, ICC 2017, fra, May 2017.

[19] R. Sanchez-Iborra and M.-D. Cano, "State of the art in LPWAN solutions for industrial IoT services," Sensors, vol. 16, no. 5, article no. 708, 2016.

[20] A. Willig, K. Matheus, and A. Wolisz, "Wireless technology in industrial networks," Proceedings of the IEEE, vol. 93, no. 6, pp. 1130-1151, 2005.

[21] D. Magrin, M. Centenaro, and L. Vangelista, "Performance evaluation of LoRa networks in a smart city scenario," in Proceedings of the 2017 IEEE International Conference on Communications, ICC 2017, fra, May 2017.

[22] J. Haxhibeqiri, A. Karaagac, F. Van den Abeele, W. Joseph, I. Moerman, and J. Hoebeke, "LoRa indoor coverage and performance in an industrial environment: Case study," in Proceedings of the 2017 22nd IEEE International Conference on Emerging Technologies and Factory Automation (ETFA), pp. 18, Limassol, September 2017.

[23] G. Margelis, R. Piechocki, D. Kaleshi, and P. Thomas, "Low Throughput Networks for the IoT: Lessons learned from industrial implementations," in Proceedings of the 2nd IEEE World Forum on Internet of Things, WF-IoT 2015, pp. 181-186, ita, December 2015.

[24] P. Neumann, J. Montavont, and T. Noel, "Indoor deployment of low-power wide area networks (LPWAN): A LoRaWAN case study," in Proceedings of the 2016 IEEE 12th International Conference on Wireless and Mobile Computing, Networking and
Communications (WiMob), pp. 1-8, New York, NY, October 2016.

[25] D. M. Hernandez, G. Peralta, L. Manero, R. Gomez, J. Bilbao, and C. Zubia, "Energy and coverage study of LPWAN schemes for Industry 4.0," in Proceedings of the 2017 IEEE International Workshop of Electronics, Control, Measurement, Signals and their Application to Mechatronics, ECMSM 2017, esp, May 2017.

[26] J. Petäjäjärvi, K. Mikhaylov, R. Yasmin, M. Hämäläinen, and J. Inatti, "Evaluation of LoRa LPWAN Technology for Indoor Remote Health and Wellbeing Monitoring," International Journal of Wireless Information Networks, vol. 24, no. 2, pp. 153-165, 2017.

[27] N. Sornin, M. Luis, T. Eirich, T. Kramp, and O. Hersent, "LoRa Alliance LoRaWAN specification," in LoRaWAN Specification, Release v1.0, 2015.

[28] C. Goursaud and J. M. Gorce, "Dedicated networks for IoT: PHY / MAC state of the art and challenges," EAI Endorsed Transactions on Internet of Things, vol. 1, no. 1, p. 150597, 2015.

[29] G. P. Hancke and V. C. Gungor, "Guest editorial special section on industrial wireless sensor networks," IEEE Transactions on Industrial Informatics, vol. 10, no. 1, pp. 762-765, 2014.

[30] L. Lo Bello, J. Akerberg, M. Gidlund, and E. Uhlemann, "Guest Editorial Special Section on New Perspectives on Wireless Communications in Automation: From Industrial Monitoring and Control to Cyber-Physical Systems," IEEE Transactions on Industrial Informatics, vol. 13, no. 3, pp. 1393-1396, 2017.

[31] Z. Iqbal, K. Kim, and H.-N. Lee, "A cooperative wireless sensor network for indoor industrial monitoring," IEEE Transactions on Industrial Informatics, vol. 13, no. 2, pp. 482-491, 2017.

[32] Y. Ai, M. Cheffena, and Q. Li, "Radio frequency measurements and capacity analysis for industrial indoor environments," in Proceedings of the 9th European Conference on Antennas and Propagation (EuCAP '15), April 2015.

[33] J. Ferrer-Coll, P. Ängskog, C. Elofsson, J. Chilo, and P. Stenumgaard, "Antenna cross correlation and ricean $\mathrm{K}$-factor measurements in indoor industrial environments at 433 and $868 \mathrm{MHz}$," Wireless Personal Communications, vol. 73, no. 3, pp. 587-593, 2013.

[34] "SX1272/73: $860 \mathrm{MHz}$ to $1020 \mathrm{MHz}$ Low Power Long Range Transceiver," http://www.semtech.com/images/datasheet/sx1272 .pdf.

[35] B. Reynders, W. Meert, and S. Pollin, "Power and spreading factor control in low power wide area networks," in Proceedings of the 2017 IEEE International Conference on Communications, ICC 2017, fra, May 2017.

[36] “E. ETSI, “300 220-1 (v2. 4.1),” Electromagnetic compatibility and Radio spectrum Matters (ERM)," 2012.

[37] “The Network Simulator - ns-3," http://www.nsnam.org/.

[38] "IEEE Standard for Information Technology - Telecommunications and Information Exchange Between Systems - Local and Metropolitan Area Networks - Specific Requirements - Part 15.4," in Wireless Medium Access Control (MAC) and Physical Layer (PHY) Specifications for Low Rate Wireless Personal Area Networks (LR-WPANs), IEEE Std., 2006.

[39] V. Rege and T. Pecorella, "A Realistic MAC and Energy Model for 802.15.4," in Proceedings of the the Workshop, pp. 79-84, Seattle, WA, USA, June 2016.

[40] A. El-Hoiydi and J. Decotignie, "WiseMAC: an ultra low power MAC protocol for the downlink of infrastructure Wireless Sensor networks," in Proceedings of the Proceedings 9th International Symposium on Computers and Communications (ISCC '04), pp. 244-251, July 2004. 
[41] D. De Guglielmo, S. Brienza, and G. Anastasi, "IEEE 802.15.4e: A survey," Computer Communications, vol. 88, pp. 1-24, 2016.

[42] P. Weber, D. Jäckle, D. Rahusen, and A. Sikora, "IPv6 over LoRaWAN $^{\mathrm{TM}}$," in Proceedings of the 3rd IEEE International Symposium on Wireless Systems within the IEEE International Conferences on Intelligent Data Acquisition and Advanced Computing Systems, IDAACS-SWS 2016, pp. 75-79, deu, September 2016. 


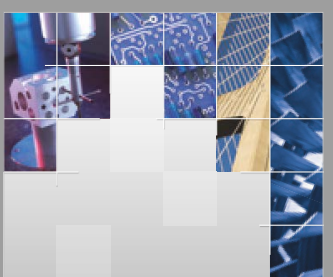

\section{Enfincering}
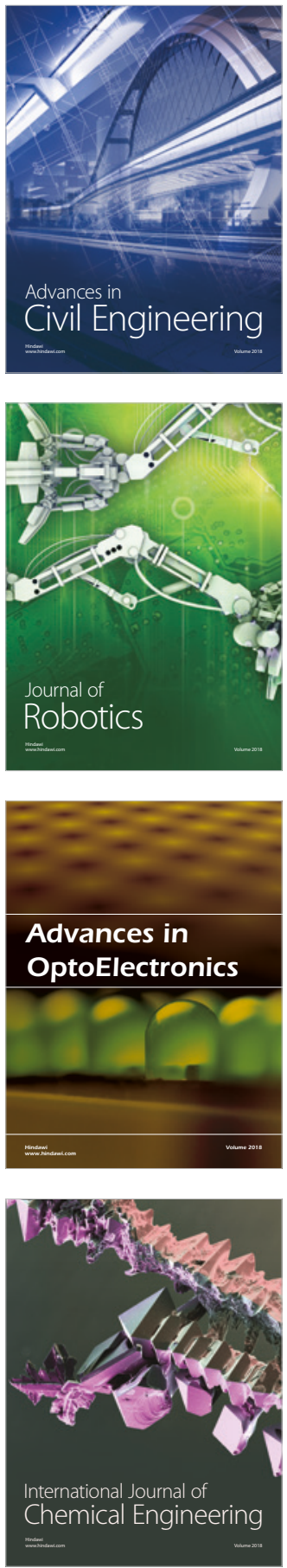

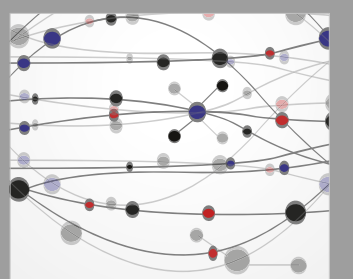

\section{Rotating \\ Machinery}

The Scientific World Journal

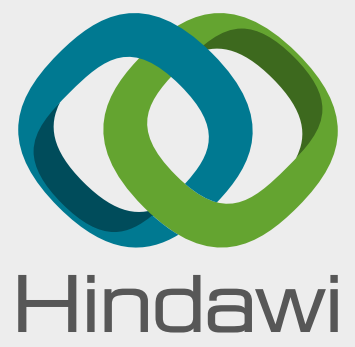

Submit your manuscripts at

www.hindawi.com
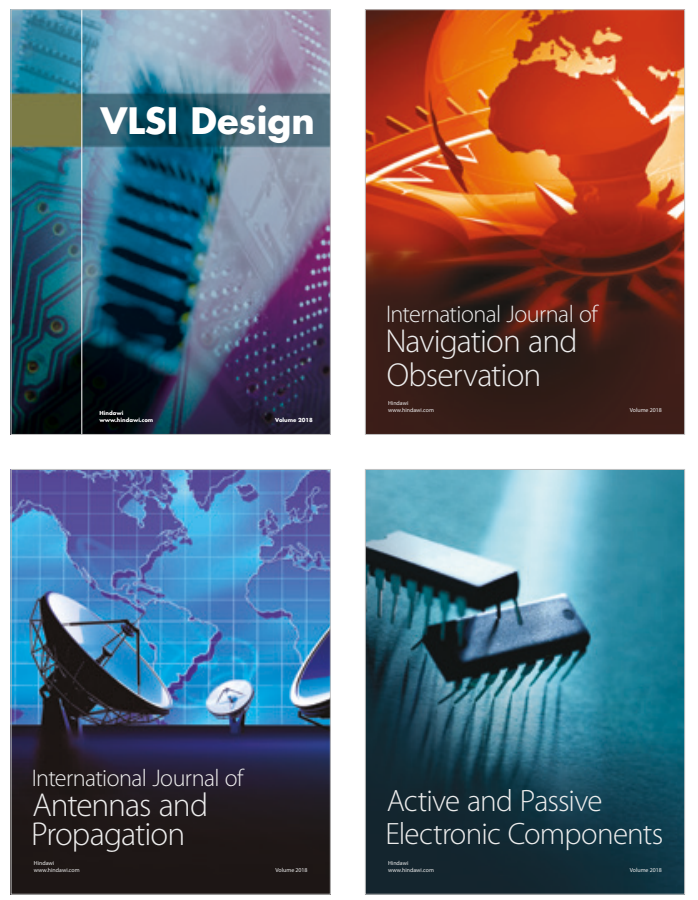
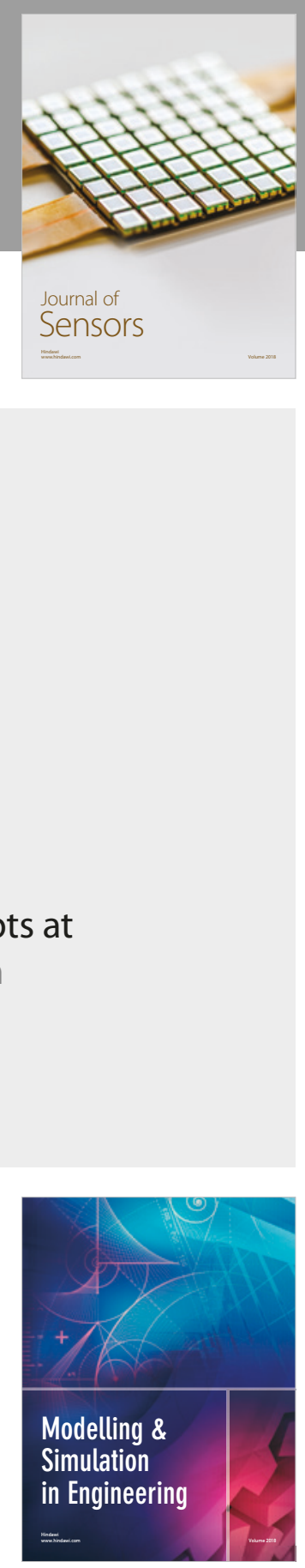

\section{Advances \\ Multimedia}
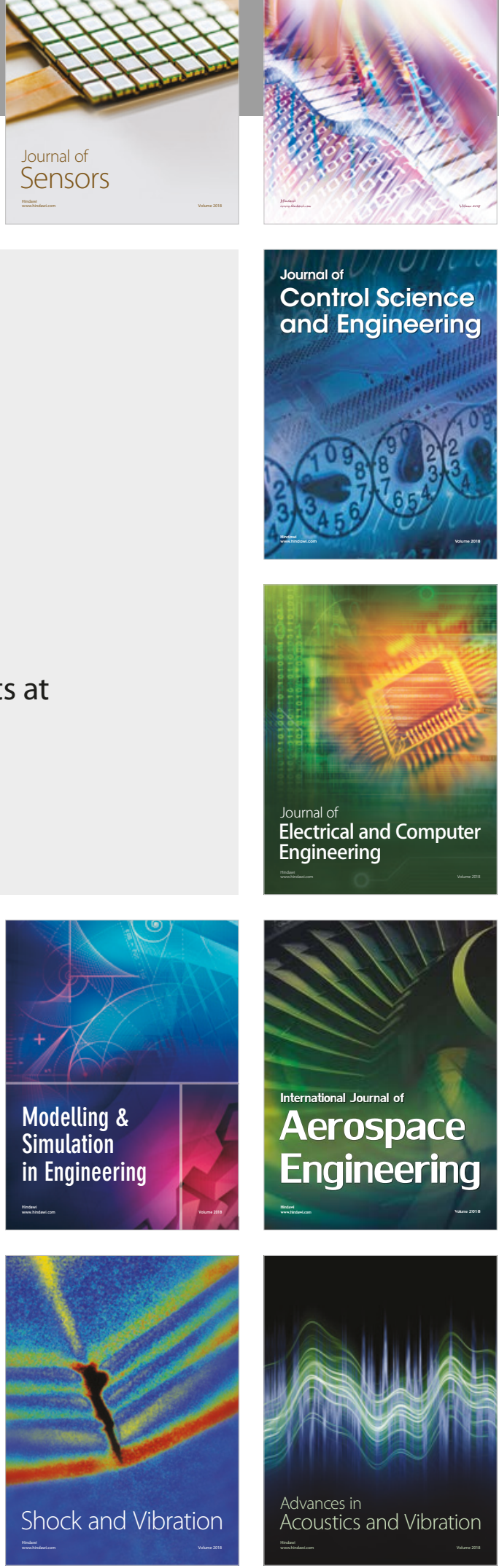